\author{
(с) Т.С. Паневин ${ }^{1,2}$, Л.И. Алексеева', Г.А. Мельниченко²
}

1 ФГБНУ «Научно-исследовательский институт ревматологии им. В.А. Насоновой», Москва, Россия ФГБУ «Национальный медицинский исследовательский центр эндокринологии», Москва, Россия

Акромегалия - это хроническое эндокринное заболевание, характеризующееся избыточной секрецией гормона роста (соматотропного гормона, СТГ), которая, в свою очередь, приводит к увеличению секреции инсулиноподобного фактора роста 1 (ИФР-1) в печени. Избыток СТГ и ИФР-1 приводит к клеточному и тканевому росту практически всех органов и систем, в том числе костно-суставного аппарата. Суставная боль при акромегалии является частым и ранним симптомом, в некоторых случаях суставные проявления могут быть одним из первых признаков, их интенсивность увеличивается с естественным течением заболевания при отсутствии лечения. В среднем признаки поражения суставов встречаются у 70\% пациентов на момент постановки диагноза. Поражение опорно-двигательного аппарата может затрагивать как осевой скелет, так и периферические суставы. Помимо артропатии, пациенты с акромегалией (как активной, так и контролируемой) чаще подвержены переломам позвонков, хотя ранее считалось, что при данном заболевании имеет место низкий риск развития остеопороза. В данном обзоре рассмотрены особенности поражения осевого скелета, периферических суставов при избыточной продукции СТГ и ИФР-1, а также ассоциация аутоиммунных ревматических заболеваний и акромегалии.

КЛЮЧЕВЫЕ СЛОВА: акромегалия; артропатия; инсулиноподобный фактор роста 1; остеоартрит; суставы; остеопороз.

\title{
RHEUMATIC MANIFESTATIONS OF ACROMEGALY
}

\section{(c) Taras S. Panevin ${ }^{1,2}$, Lyudmila I. Alekseeva', Galina A. Melnichenko²}

'V.A. Nasonova Research Institute of Rheumatology, Moscow, Russia

${ }^{2}$ Endocrinology Research Centre, Moscow, Russia

Acromegaly is a chronic endocrine disease characterized by excessive secretion of growth hormone (GH), which, in turn, leads to increased insulin-like growth factor 1 (IGF-1) secretion by the liver. GH and IGF-1 excess leads to excessive cell and tissue growth, including the osteoarticular apparatus. Joint pain in acromegaly is a frequent and early symptom. In some cases, joint manifestations can be one of the first signs of acromegaly and their intensity increases with duration of the active disease. Estimated prevalence of joint damage signs is around $70 \%$ of patients at the time of diagnosis of acromegaly. Musculoskeletal system alterations can manifest either in axial skeleton and peripheral joints. Besides arthropathy, patients with acromegaly (both active and controlled) are more prone to vertebral fractures, although it was previously thought that acromegaly has a low risk of osteoporosis. In this article, we review features of damage to the axial skeleton, peripheral joints in the setting of excessive GH and IGF-1 production, as well as the association of autoimmune rheumatic diseases and acromegaly.

KEYWORDS: acromegaly; arthropathy; insulin-like growth factor 1; osteoarthritis; joints; osteoporosis

\section{ВВЕДЕНИЕ}

Акромегалия - это хроническое эндокринное заболевание, характеризующееся избыточной секрецией гормона роста (соматотропного гормона, СТГ), которая, в свою очередь, приводит к увеличению секреции инсулиноподобного фактора роста 1 (ИФР-1) в печени [1]. Мишенями для данных гормонов являются большинство клеток нашего организма. Избыток СТГ (в подавляющем большинстве случаев обусловленный гормон-продуцирующей аденомой гипофиза - соматотропиномой) и ИФР-1 приводит к клеточному и тканевому росту практически всех органов и систем, в том числе костно-суставного аппарата. Распространенность акромегалии составляет 4,6 случая на 1 млн населения, а заболеваемость - 116,9 новых случая на 1 млн ежегодно [2]. Характерными симптомами данного заболевания являются изменение внешности, в первую очередь «огрубение» черт лица, увеличение размеров конечностей, изменение тембра голоса, а также общая усталость, головные боли и артралгии, парестезии и повышенная потливость [3]. Акромегалия характеризуется медленным темпом развития клинических признаков, что зачастую приводит к поздней постановке диагноза и может являться причиной ранней инвалидизации.

Суставная боль является частым и ранним симптомом, в некоторых случаях суставные проявления могут быть одним из первых признаков акромегалии, их интен- 
сивность увеличивается с естественным течением заболевания при отсутствии лечения. Среднее время от начала заболевания до первых клинических проявлений со стороны опорно-двигательного аппарата составляет около 10 лет [4]. Артропатия при акромегалии впервые описана P. Marie в 1886 г. [5]. Поражение опорно-двигательного аппарата вызывает значительное ухудшение качества жизни даже при достижении длительной устойчивой ремиссии основного заболевания. Риск развития остеоартрита у пациентов с акромегалией увеличен в 4-12 раз по сравнению с общей популяцией, в том числе в молодом возрасте [6].

Доступными методами лечения акромегалии являются транссфеноидальная аденомэктомия, лучевая терапия, а также медикаментозная терапия с использованием аналогов соматостатина (AC) и пэгвисоманта аналога СТГ, модифицированного молекулами полиэтиленгликоля. Однако вопрос лечения акромегалической артропатии остается актуальным и нерешенным [7].

Нами проведен анализ публикаций к июню 2019 г. в системе медицинской литературы в библиографических базах Medline и Cochrane Collaboration за период с 1990 по 2019 г. Поисковый запрос формировался по следующим ключевым словам: акромегалия, акромегалия и артропатия, акромегалия и суставы, ревматические нарушения и акромегалия.

В среднем признаки поражения суставов встречаются у 70\% пациентов на момент постановки диагноза акромегалии [5]. Поражение опорно-двигательного аппарата может затрагивать как осевой скелет (преимущественно в поясничном отделе позвоночника), так и периферические суставы (преимущественно коленный, тазобедренный, плечевой, лучезапястный, мелкие суставы кистей) [8]. По результатам исследований, рентгенологические признаки артропатии хотя бы в одном суставе обнаруживаются у 99\% пациентов, наиболее часто в позвоночнике и тазобедренных суставах. Отмечено несоответствие выраженности болевого синдрома и функциональной недостаточности рентгенологической картине [6].

\section{ПАТОГЕНЕЗ}

Патогенетические изменения при акромегалической артропатии связаны с избытком СТГ и ИФР-1, вторичные нарушения на начальных этапах обусловлены эндокринными изменениями с последующим развитием дегенеративных процессов. Отмечено, что у пациентов с полиморфизмом гена рецептора СТГ, а именно делецией экзона 3 (d3-GHR), которая приводит к повышенной реакции на воздействие СТГ, наблюдается повышенная распространенность необратимых осложнений акромегалии, таких как артропатия, долихоколон и аденоматозные полипы толстой кишки [9].

Артропатия развивается, прежде всего, из-за нарушенного гомеостаза матрикса суставного хряща, который регулируется несколькими факторами роста, и ИФР-1 является наиболее важным стимулятором роста хрящевой ткани [10]. Помимо анаболического действия на нормальный суставной хрящ, ИФР-1 продемонстрировал свою эффективность в восстановлении поврежденного хряща на животных моделях [11].
На ранних стадиях заболевания избыток СТГ и ИФР-1 приводит к увеличению синтеза протеогликана и гликозаминогликана суставными хондроцитами, усилению клеточной репликации [12], способствует росту хрящевой и периартикулярных тканей, увеличению толщины суставного хряща. Разрастания этих тканей приводят к ограничению амплитуды движений в суставе, а гипертрофированные связки вызывают нарушение стабильности сустава. Рентгенологически данная стадия характеризуется расширением суставного пространства и околосуставной гипертрофией мягких тканей. При адекватном биохимическом контроле акромегалии данные изменения могут носить, по крайней мере частично, обратимый характер [5].

Указанные процессы приводят к увеличению капсулы сустава, расширению суставного пространства и, как следствие, изменению нормальной геометрии сустава, что в последующем способствует повторным внутрисуставным микротравмам и избыточным репаративным реакциям с образованием субхондральных кист, остеофитов, приводящих к ухудшению функционального состояния сустава. Рентгенологические изменения на этой стадии характеризуются сужением суставных пространств и другими дегенеративными изменениями. На данном этапе артропатия имеет схожие морфологические проявления с остеоартритом и становится необратимой, при этом достижение нормального уровня ИФР-1 будет иметь небольшую эффективность в улучшении клинического статуса [13].

На сегодняшний день дискутабельным остается вопрос: является ли регрессия размеров суставного пространства признаком остеоартрита или отражением продолжающейся нормализации гипертрофированного хряща после индукции ремиссии. В некоторых краткосрочных ультразвуковых исследованиях показано сужение суставной щели после достижения биохимического контроля акромегалии [14].

В целом при акромегалии рентгенографическая картина характеризуется наличием выраженного остеофитоза, часто в сочетании с нормальной или даже расширенной суставной щелью (рис. 1) [15]. Некоторые

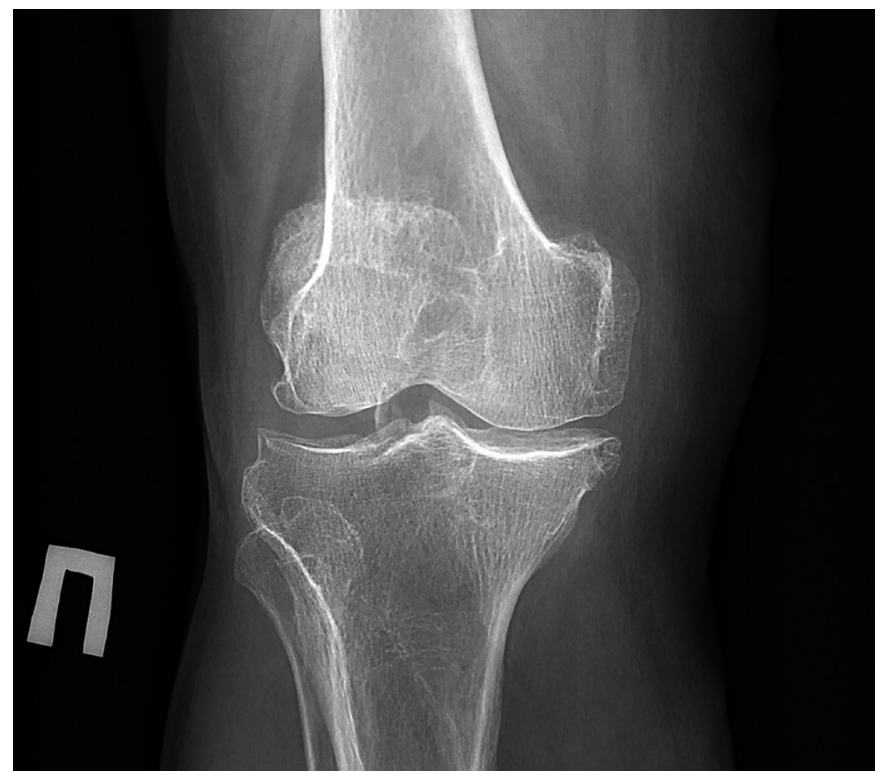

Рис. 1. Расширение суставной щели на фоне выраженного остеофитоза у больного акромегалией 1947 г.р. 
исследователи принципиально выделяют два рентгенологических типа артропатии: остеофитоз в сочетании с сохраненной или расширенной суставной щелью и, более редкий, с наличием или отсутствием остеофитов и сужением суставной щели. В настоящее время процесс сужения суставной щели при акромегалии до конца не изучен, и неизвестно, является ли данный рентгенографический признак конечной стадией акромегалической артропатии, когда избыток СТГ/ИФР-1 превысил некий критический порог, или отдельной ее формой [16].

\section{ПОРАЖЕНИЕ ПЕРИФЕРИЧЕСКИХ СУСТАВОВ}

Для акромегалии характерно поражение как крупных, так и мелких периферических суставов. Наиболее часто поражаются суставы нижних конечностей (коленный и тазобедренный). Распространенность клинического и рентгенологического поражения тазобедренного сустава, мелких суставов кистей и коленного сустава достигает $80 \%$.

Kropf и соавт. обследовали 71 пациента с акромегалией, выделив две группы по тяжести течения артропатии в зависимости от наличия превышения нормы уровня СТГ и ИФР-1 (21 мужчина (29,5\%) и 50 женщин (70,5\%) со средним возрастом 49,5 \pm 14,5 лет). Оценивалось поражение коленных, тазобедренных и плечевых суставов. Артропатия наблюдалась у 40 пациентов (56\%), была отмечена статистически значимая связь артропатии с увеличением индекса массы тела (ИМТ), возрастом на момент постановки диагноза акромегалии и женским полом, которые, в свою очередь, признаны факторами риска развития остеоартрита. Не было обнаружено связи между наличием артропатии и концентрацией СТГ и ИФР-1 в крови, а также длительностью существования высоких показателей СТГ и ИФР-1 до начала лечения акромегалии. Более тяжелая артропатия развивалась при более высоком ИМТ и более низком уровне ИФР-1 [17].

Примечательно, что пациенты с акромегалией в целом имеют меньшее снижение функциональной активности за счет гипертрофированного суставного хряща, чем пациенты с первичным генерализованным остеоартритом, несмотря на большую распространенность и выраженность остеофитов [15]. Отмечено более частое выявление по данным рентгенографии хондрокальциноза, однако частота приступов псевдоподагры соответствует общепопуляционной. Следует отметить, что в случае, если акромегалия является составляющим компонентом синдрома множественных эндокринных неоплазий 1 типа (МЭН-1), наличие хондрокальциноза и псевдоподагрических приступов может быть также обусловлено первичным гиперпаратиреозом, встречающимся в 90\% случаев МЭН-1 [18].

В исследовании Claessen и соавт. оценивались MPизменения коленного сустава для изучения структурных особенностей акромегалической артропатии по сравнению с первичным остеоартритом (OA). В это исследование были включены 10 пациентов с активной акромегалией, 16 пациентов с биологической ремиссией акромегалии и 25 пациентов с первичным ОА. У пациентов с акромегалией наблюдались большая толщина суставного хряща, меньшее количество субхондральных кист и менее выраженный отек костного мозга. Отмечена большая толщина хряща у пациентов с активной акромегалией, чем у пациентов в ремиссии [19].

Пациенты с акромегалией имеют лучшие показатели индекса WOMAC (отражает выраженность боли, скованности и функциональных нарушений), чем пациенты с первичным ОА, несмотря на выраженный остеофитоз, что, вероятно, обусловлено сохранением нормальных размеров суставной щели пораженных суставов. Отмечена меньшая потребность в эндопротезировании суставов в целом, и особенно тазобедренного сустава, чем у пациентов с первичным ОА [15].

Дефигурация периферических суставов чаще всего обусловлена утолщением синовиальной оболочки и периартикулярных тканей, а не наличием выпота [20]. В отсутствие специфической системы классификации для акромегалической артропатии предлагается использование классификационных критериев OARSI (Международного общества по изучению остеоартроза) ввиду возможности раздельного учета наличия остеофитов и сужения суставной щели (ССЩ) [15]. Однако важным ограничением этих неспецифических классификаций является отсутствие в них признака, с наибольшей вероятностью отражающего тяжесть акромегалической артропатии, - расширения суставной щели.

Nezu и коллеги проанализировали влияние трансназальной аденомэктомии (ТНАЭ) на течение периферической артропатии у 16 пациентов с акромегалией (68,9\% женщин, средний возраст 58 лет) с помощью МРТ коленного сустава. Первоначально утолщение синовиальной оболочки, отек костного мозга, повреждение связок и мениска были выявлены у 22,7\%, 22,7\%, 4,7\% и 59,1\% пациентов соответственно. Через 2 мес после оперативного лечения отмечено уменьшение болевого синдрома в коленном суставе, у 66,7\% уменьшились выраженность утолщения синовиальной оболочки и отек костного мозга, однако не изменялась структура ранее поврежденного мениска [21]. Отсутствие улучшения МР-картины мениска может быть одной из причин сохранения боли и прогрессирования поражения коленного сустава после достижения ремиссии [22]. Однако данное исследование ограничено небольшим количеством пациентов и коротким интервалом динамической оценки.

ССЩ ассоциируется с более частым наличием жалоб как со стороны коленного, так и тазобедренного сустава [15]. По данным исследования Claessen и колл., встречаемость ССЩ при акромегалии составляет 10-15\% [23]. В тазобедренном суставе активность акромегалии (более высокие уровни СТГ/ИФР-1 при постановке диагноза) положительно коррелировала с ССЩ, послеоперационное улучшение достигалось реже. Таким образом, ось СТГ/ИФР-1, вероятно, играет роль не только на ранней, но и на поздней стадии акромегалической артропатии. Однако в коленном суставе выраженность ССЩ была ассоциирована с хирургическим вмешательством, например, менискэктомией, но не с активностью акромегалии.

Высокая распространенность ССЩ у пожилых пациентов и женщин согласуется с предыдущими наблюдениями по первичному ОА. Хорошо известно, что дисплазии суставов, переломы суставных поверхностей, 
разрывы менисков и повреждения связок увеличивают нестабильность суставов, а также часто предшествуют развитию ОА [24], поэтому связь ССЩ в коленном суставе с предшествующим избытком СТГ сомнительна.

Интересным наблюдением является высокая распространенность ССЩ у пациентов, получавших $A C$, в отличие от относительно низкой распространенности у пациентов, достигших ремиссии после ТНАЭ. Данная корреляция прослеживались только для тазобедренного сустава. Длительность лечения АС была ассоциирована с выраженностью сужения щели и, особенно, остеофитозом. Данное явление может быть объяснено, во-первых, более тяжелым течением заболевания у пациентов, получавших AC, так как во время их применения могут сохраняться незначительные превышения секреции СТГ и ИФР-1 [25]. Так, сообщалось о менее благоприятном исходе у пациентов, получавших АС, в отношении качества жизни и диастолической функции сердца [26]. С другой стороны, АС, вероятно, могут оказывать прямое, независимое от ИФР-1 влияние на структуру сустава. Имеются данные о прямом ингибирующем локальном воздействии соматостатина на хрящ [27], и, кроме того, соматостатиновые рецепторы (СР) обнаружены на остеобластах [28]. Предполагается, что у некоторых пациентов терапия AC может оказывать эффект только на ИФР-1, в то время как влияние СТГ на хрящ сохраняется. Однако, согласно результатам немногочисленных исследований, на фоне терапии пэгвисомантом, который исключает влияние СТГ на суставной хрящ, не выявлено улучшения суставного синдрома.

Так, в рамках наблюдательного немецкого исследования (GPOS) у 131 пациента с акромегалией на фоне терапии пэгвисомантом в течение 1 года (средняя доза $16,5 \pm 8,7$ мг/сут) показано отсутствие уменьшения суставной боли, согласно результатам опросника «PatientAssessed Acromegaly Symptom Questionnaire» (PASQ) [29]. Исследование Neggers и колл. также не показало динамики суставной боли по PASQ в течение 32 нед на фоне терапии пэгвисомантом в дозе 40 мг/сут у 20 больных с достижением биохимического контроля акромегалии [30].

Для оценки степени утолщения хряща и мониторинга эффективности лечения АС может быть полезно УЗИ суставов. Анализ, проведенный у пациентов с неактивной акромегалией в течение 2 лет, показал, что длительный период лечения улучшает течение артропатии коленных суставов. Так, подавление циркулирующих уровней СТГ и ИФР-1 на фоне 12-месячной терапии октреотидом пролонгированного действия сопровождалось значительным уменьшением толщины суставного хряща как несущих, так и не несущих суставов независимо от продолжительности заболевания. Снижение уровней СТГ и ИФР-1 коррелирует с ССЩ лучезапястного и коленного сустава [14]. Терапия октреотидом короткого действия в дозе 0,3-0,6 мг/сут в течение 6 мес значительно снижала толщину суставного хряща в плечевом, лучезапястном и левом коленном суставах [31], а 12-месячное лечение ланреотидом привело к значительному сужению суставной щели как опорных, так и не опорных суставов [32]. Тем не менее ни в одном исследовании не наблюдалось полного регресса утолщения суставного хряща.

Характерные рентгенологические изменения при акромегалической артропатии сохраняются у большин- ства пациентов и после достижения биохимического контроля заболевания [6]. Вместе с тем у меньшей части пациентов отмечаются противоположные изменения (ССЩ) [23] с более выраженными суставными симптомами и бо́льшим стажем акромегалии. Несмотря на длительный биохимический контроль заболевания, более чем у 70\% пациентов остеофитоз прогрессирует [33]. Таким образом, фенотип акромегалической артропатии с ССЩ встречается значительно реже. Пациенты с ССЩ имеют больше суставных жалоб, чем пациенты с нормальным или увеличенным размером суставной щели [34].

\section{ПОРАЖЕНИЕ ОСЕВОГО СКЕЛЕТА}

Боль в спине является частым симптомом акромегалии и присутствует примерно у 50\% пациентов [35]. По данным клинического и рентгенологического обследования, аксиальная артропатия диагностируется у около 60\% на момент постановки диагноза акромегалии [8]. Длительность акромегалии коррелирует с распространенностью поражения осевого скелета. Наиболее частой жалобой являются диффузные тупые боли в поясничной области. Боль не распространяется на нижние конечности, и часто присутствует как в покое, так и при физической нагрузке. При поражении позвоночника, так же как и при поражении периферических суставов, сохраняется нормальный объем движений, что, возможно, обусловлено гипертрофией межпозвоночных дисков, суставных хрящей и растяжением связок [20].

Рентгенологическая картина характеризуется наличием остеофитов, особенно в передних отделах тел позвонков, у пациентов с акромегалией часто наблюдается расширение межпозвонкового пространства. Нередко у данных пациентов встречается оссификация передней продольной связки (рис. 2 и 3). Процесс окостене-

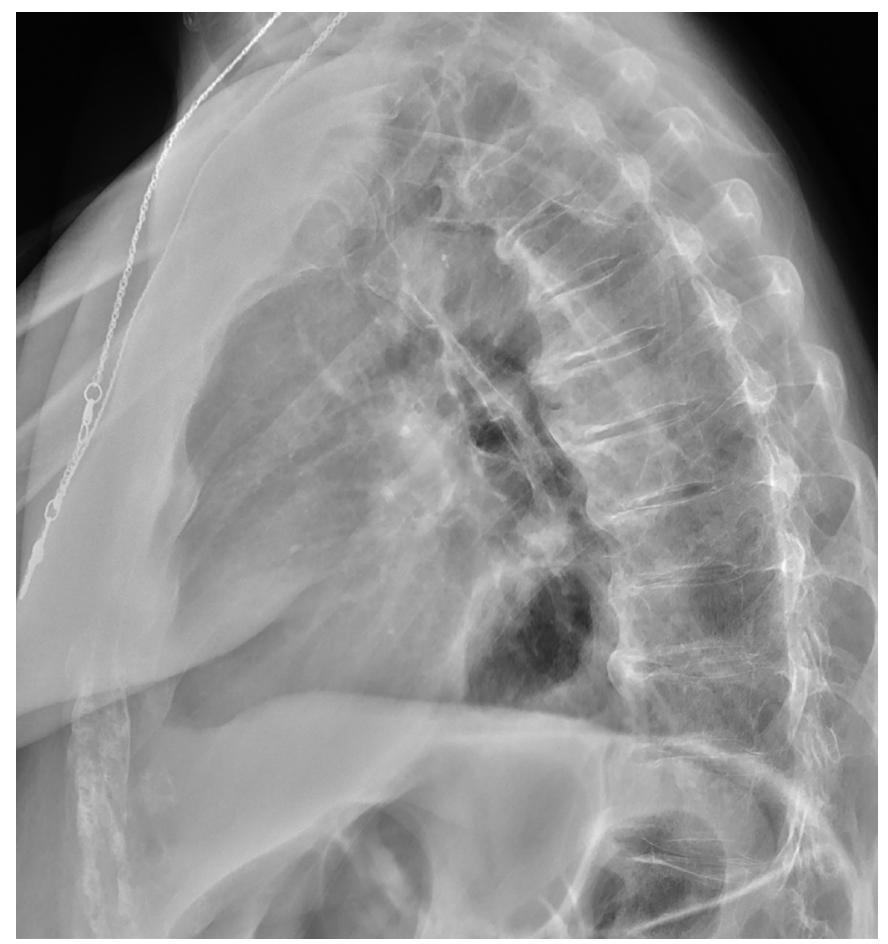

Рис. 2. Обызвествление передней продольной связки и уменьшение дискового пространства у пациента 1954 г.р. с акромегалией. 


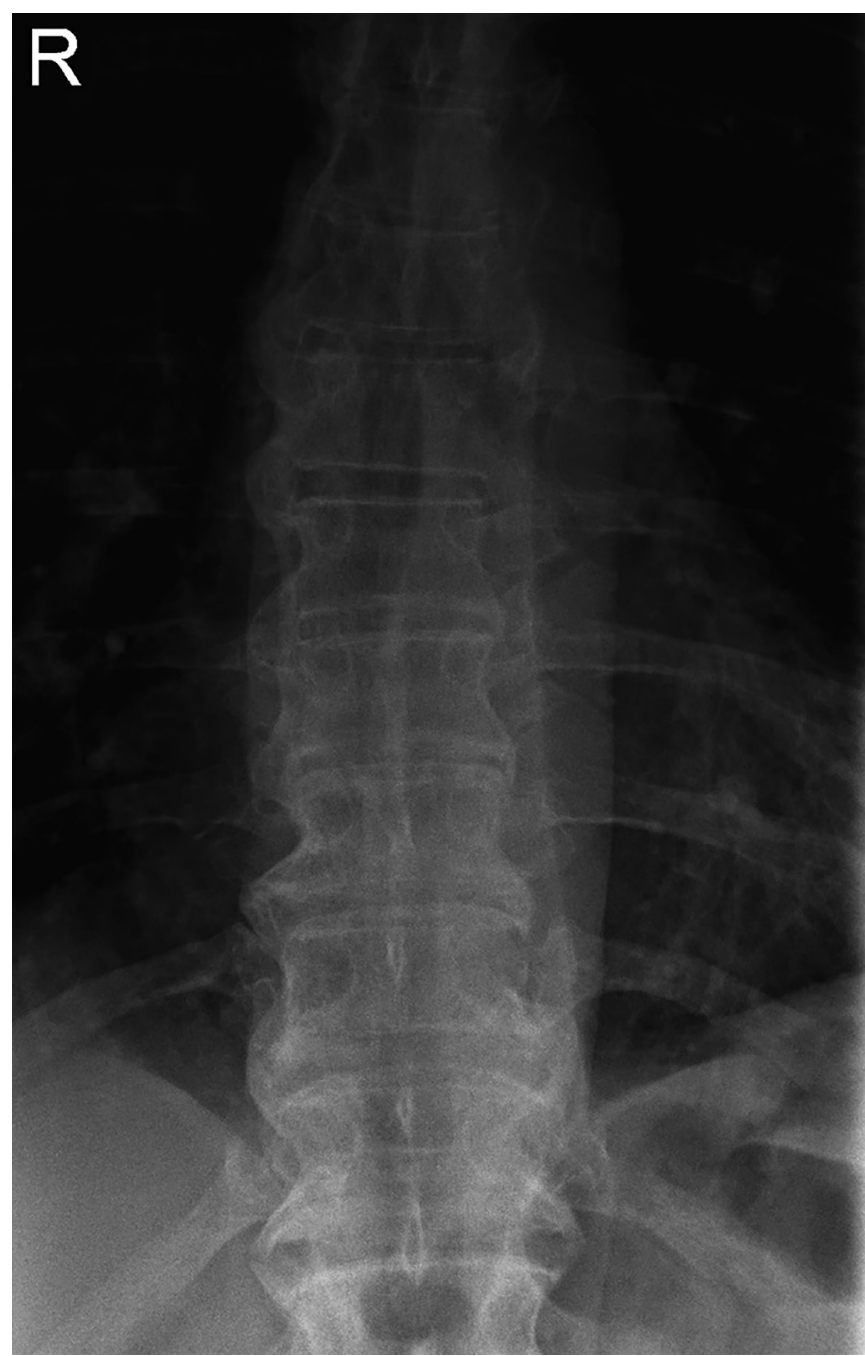

Рис. 3. Выраженное обызвествление передней продольной связки грудного и поясничного отделов позвоночника преимущественно справа у пациента 1960 г.р. с акромегалией.

ния может полностью захватывать межпозвонковую щель, и иметь одинаковую рентгенологическую картину с диффузным идиопатическим гиперостозом (болезнью Форестье, БФР). При акромегалии данное состояние встречается В 15-20\% случаев, с преимущественной локализацией в нижнем грудном и поясничном отделах позвоночника [8]. В популяции БФР наиболее часто ассоциируется с метаболическим синдромом, а также с сахарным диабетом и подагрой [36].

В исследовании Scarpa и соавт. [8], которое включало 54 пациента с активной акромегалией (27 мужчин, 27 женщин; возрастной диапазон 21-69 лет) и 54 человека группы контроля, аксиальная артропатия была обнаружена у 28 пациентов (52\%) и у 12 в группе контроля (22\%). Ограничение подвижности позвоночника наблюдалось у 30 пациентов (56\%) и 10 в группе контроля (18\%), увеличение диаметра $L_{\|}$позвонка наблюдалось у 34 пациентов (63\%) и ни у одного из контрольных пациентов. Сужение и расширение междискового пространства $\mathrm{L}_{\|}-\mathrm{L}_{\text {III }}$ были обнаружены у 20 (37\%) и 7 (13\%) пациентов соответственно. Диффузный идиопатический гиперостоз скелета был обнаружен у 11 пациентов (20\%) и ни у одного из группы контроля. Длительность акромегалии положительно коррелировала с высотой тела $\mathrm{L}_{\|}$или размером межпозвонкового пространства. Тяжесть БФР коррелировала с базальным и пиковым уровнями глюкозы после перорального глюкозотолерантного теста, но не с длительностью и тяжестью акромегалии.

Отсутствие значимой корреляции между наличием артропатии и возрастом пациентов, возрастом начала акромегалии, а также уровнями СТГ и ИФР-1 позволяет предположить, что поражение позвоночника может иметь гормон-независимый характер. Однако положительная корреляция между размером междискового пространства и высотой тела позвонка с продолжительностью заболевания, а также переднезадним диаметром тела позвонка может говорить о том, что хроническое воздействие повышенного СТГ и ИФР-1 влияет на физиологический баланс позвоночника, вызывая непропорциональное увеличение диаметра позвонков и рост тканей диска. Данные изменения в итоге могут вызвать нарушение трофики как хряща, так и суставного диска, приводя к дегенерации мягких тканей [37].

Таким образом, несколько факторов могут способствовать возникновению аксиальной артропатии при акромегалии, каждый из которых имеет особое значение, но сам по себе недостаточен в качестве обязательного маркера ее развития. Эндокринные факторы, такие как степень и продолжительность воздействия избытка СТГ и ИФР-1, вероятно, действуют в совокупности с местными нарушениями биомеханики суставов. Обусловленное взаимодействием множества факторов и индивидуальных анатомических характеристик поражение позвоночника у пациентов с акромегалией характеризуется вариабельностью, включая формы преимущественно функциональные и, следовательно, потенциально обратимые [38] и формы, характеризуемые серьезными морфологическими и структурными изменениями, с неблагоприятным прогнозом в плане регресса после достижения ремиссии акромегалии.

Остеопороз и патологические переломы. Помимо артропатии, больные акромегалией (как активной, так и контролируемой) чаще подвержены переломам позвонков, хотя ранее считалось, что данные пациенты имеют низкий риск развития остеопороза, поскольку минеральная плотность кости не снижается и даже повышается в результате анаболического действия СТГ и ИФР-1 [39]. Однако из-за вышеупомянутых структурных изменений позвоночника результаты денситометрии могут быть завышены [40]. Наиболее часто имеют место патологические переломы в грудном отделе позвоночника [16].

Два независимых проспективных исследования показали, что пациенты с акромегалией имеют высокий риск переломов тел позвонков $[16,41]$. Переломы позвонков были отмечены у 42\% пациентов при 3-летнем периоде наблюдения, их распространенность зависела от длительности активной фазы заболевания.

Высокая распространенность переломов позвонков отмечена у женщин в постменопаузе и у мужчин с акромегалией, вне зависимости от показателей минеральной плотности костной ткани (МПК). Частота переломов позвонков была ассоциирована с активностью акромегалии и наличием таких нарушений, как гипогонадизм и сахарный диабет $[42,43]$. Считается, что избыток СТГ воздействует на качество костной ткани, а не на объем [39]. Примечательно преимущественное анаболическое влияние СТГ на кортикальную костную ткань, тогда как 
трабекулярная микроархитектоника, напротив, ухудшается под действием избыточной концентрации СТГ [44]. Не выявлено корреляции между длительностью поддержания нормального уровня СТГ и ИФР-1 и МПК, а также маркерами костного обмена. По результатам исследований, повторные переломы позвонков наблюдались у $20 \%$ пациентов с медикаментозной или хирургически-контролируемой ремиссией акромегалии [45].

Уровень половых гормонов пациентов также влияет на МПК. Нередко встречающийся у данных пациентов гипогонадизм способствует снижению МПК преимущественно в поясничном отделе позвоночника [46], в то время как влияние на шейку бедра менее очевидно [42]. Следует отметить, что нейрохирургическое и радиохирургическое лечение может привести к развитию гипопитуитаризма, что может повлиять на минеральную плотность и архитектонику костной ткани [47].

Ряд авторов предлагают проводить боковое рентгенологическое исследование грудного и поясничного отдела позвоночника в момент постановки диагноза и при динамическом наблюдением с интервалом 12-24 мес для выявления переломов позвонков даже при условии достижения биохимической ремиссии [45]. Для точного выявления переломов позвонков целесообразно использовать радиологический морфометрический подход [48].

\section{АУТОИММУННАЯ РЕВМАТИЧЕСКАЯ ПАТОЛОГИЯ ПРИ АКРОМЕГАЛИИ}

Боль в суставах является преобладающим симптомом при акромегалии, как правило, ассоциирована с физической активностью и носит преходящий характер [20]. Припухание суставов и скованность могут быть симптомами как акромегалии, так и ревматического заболевания. Боль после отдыха может иметь место у 20\% пациентов с акромегалией [35]. По результатам исследований отмечена более редкая встречаемость ревматической патологии аутоиммунного характера у пациентов с акромегалией в сравнении с общей популяцией [49].

В литературе встречается ряд описаний клинических случаев сочетания акромегалической артропатии и ревматического заболевания, подчеркивая необходимость дифференциальной диагностики данных состояний, а также выявление их сочетания. Ozcakar и соавт. [50] представили 63-летнего пациента с сочетанием серопозитивного ревматоидного артрита (РА), диагностированного через 18 лет от начала акромегалии и акромегалической артропатии. В другом обзоре сообщается о двух пациентах с жалобами на двустороннюю симметричную боль, отечность и утреннюю скованность в суставах кистей после радикального достижения ремиссии акромегалии. После лабораторного исключения рецидива акромегалии был диагностирован РА, суставные симптомы регрессировали после назначения базисной противовоспалительной терапии [51]. Read и колл. [52] сообщили о 57-летней женщине с серопозитивным РА, суставной синдром при котором изначально рассматривался как проявления акромегалии на протяжении 12 лет, акцентируется внимание на том, что гиперпродукция СТГ при акромегалии может замедлять катаболические изменения хрящевой и костной ткани при РА. Имеется описание
РА у пациентки 70-летнего возраста с сохранением болей в суставах и утренней скованности после оперативного лечения акромегалии, симптомы также регрессировали после назначения метотрексата [53].

Описаны и случаи ведения пациентов с недиагностированной акромегалической артропатией как ревматического заболевания. В отчете Nachtigall первыми симптомами акромегалии были артрит и головная боль, проводилось лечение гидроксихлорохином и глюкокортикоидами в течение четырех лет с диагнозом РА при сохранении суставных симптомов. В последующем обнаружена макроаденома гипофиза, пациент перенес транссфеноидальную аденомэктомию, которая привела к уменьшению боли в суставах и головной боли [54].

Обширное исследование ревматических заболеваний у пациентов с акромегалией было выполнено Podgorski и др. Серопозитивный РА был зарегистрирован у одного из 45 пациентов, у двух пациентов была ревматическая полимиалгия, и у одного пациента - палиндромный ревматизм [35].

В исследовании Oruk и соавт. [49] был сделан акцент на ревматические заболевания у пациентов с акромегалией, получавших АС. Акромегалическая артропатия отмечалась у 62,5\% пациентов, БФ - у шести пациентов (15\%). По результатам лабораторных исследований выявлено повышение титра антинуклеарного фактора у 4 пациентов (3 нуклеолярного и 1 гомогенного типа свечения), позитивность ревматоидного фактора у четыpex, а также наличие антител к циклическому цитруллинированному пептиду у одного пациента. Диагнозы РА, анкилозирующий спондилит и недифференцированное заболевание соединительной ткани были поставлены 3 из 40 пациентов. На фоне соответствующего лечения отмечалась регрессия симптоматики.

Данные исследования показывают, что суставные жалобы у пациентов с акромегалией чаще вызваны акромегалической артропатией. Кроме того, у отдельных пациентов с акромегалией могут выявляться сопутствующие ревматические заболевания, которые требует дифференциальной диагностики в рамках суставного синдрома.

Синдром Рейно (СР) характеризуется преходящими эпизодами вазоспазма пальцев кистей под действием холодной температуры или эмоционального стресса. Данное клиническое состояние проявляется последовательными изменениями окраски кожи пальцев кистей и/или стоп, которые отражают разные фазы состояния: побеление (вазоконстрикция), посинение (гипоксия) и покраснение (реактивная гиперемия по завершении вазоспазма), так называемый трехфазный СР. При акромегалии СР встречается примерно в 1/3 случаев [55]. Основное место в дифференциальной диагностике СР отводится капилляроскопии ногтевого ложа, которая позволяет выявить структурные изменения капилляров, характерные для системных заболеваний соединительной ткани (в первую очередь для системной склеродермии) [56]. По данным исследований с использованием капилляроскопии, продемонстрировано уменьшение длины и количества капилляров у пациентов с активной фазой акромегалии, а также увеличение числа извитых капилляров по сравнению с пациентами в ремиссии и группой контроля. Эти изменения коррелируют с активностью заболевания, являются частично 
обратимыми, присутствуют как у пациентов с диабетом и без диабета, так и у пациентов с артериальной гипертензией и нормальным артериальным давлением [57]. Данные о динамике течения СР после аденомэктомии в современной литературе отсутствуют.

Помимо указанных состояний, следует отметить, что при акромегалии встречается синдром запястного (карпального) канала (30-50\% пациентов), часто он бывает двусторонним. Компрессия и гипертрофия срединного нерва является патогенетической основой данного состояния и приводит к характерной клинической картине синдрома в виде неврологической симптоматики и в последующем атрофии ладонных мышц. В исследовании Миллера и колл. 58 пациентам с акромегалией 17,2\% ранее проводилась кистевая туннельная декомпрессия и 39,7\% имели признаки данного состояния при постановке диагноза [58]. Субклинический вариант, характеризующийся только изменениями по результатам нейрографии, имел место у $81 \%$ пациентов. Несколько исследований подтвердили, что улучшение симптомов происходит при снижении секреции СТГ после радикального лечения: в исследовании Kameyama после аденомэктомии или лучевой терапии нервная проводимость нормализовалась у 26\% пациентов [59].

Поскольку при акромегалической артропатии часто наблюдается образование остеофитов без других дегенеративных изменений, формирование выраженных костных разрастаний может привести к синдрому щелкающего пальца, хотя в целом данное состояние при акромегалии встречается редко [60].

\section{ЗАКЛЮЧЕНИЕ}

Таким образом, акромегалическая артропатия является прогрессирующим заболеванием суставов, лечение которого сводится не только к достижению ремиссии акромегалии. При вовлечении суставов целесообразно быстрое достижение биохимического контроля акроме- галии, предпочтительно путем хирургического лечения. Следует подчеркнуть, что у ряда пациентов с акромегалической артропатией, даже несмотря на биохимическую ремиссию, прогрессирование поражения суставов продолжается. Учитывая общие конечные патогенетические пути и схожие рентгенологические изменения с первичным ОА, вероятно, дальнейшее лечение поражения суставов должно быть аналогичным терапии ОА и включать приём симптоматических препаратов замедленного действия (хондропротекторов), курсовой прием нестероидных противовоспалительных препаратов, локальную поверхностную и инъекционную терапию. Эффективность применения данных препаратов у пациентов с акромегалической артропатией не изучалась и требует проведения дальнейших исследований. Необходимо отметить более высокую распространенность переломов тел позвонков при акромегалии, по сравнению с общей популяцией, при нормальной или повышенной МПК.

Хотя акромегалическая артропатия и дегенеративные поражения суставов часто встречаются у пациентов с акромегалией, системные воспалительные ревматические заболевания тоже могут сосуществовать с акромегалией, особенно у пациентов с сохранением суставных симптомов после достижения биохимической ремиссии основного заболевания. В связи с этим при наличии жалоб со стороны суставов необходима не только дифференциальная диагностика, но и совместное наблюдение таких пациентов эндокринологом и ревматологом.

\section{ДОПОЛНИТЕЛЬНАЯ ИНФОРМАЦИЯ}

Конфликт интересов. Авторы заявляют об отсутствии конфликта интересов в ходе написания данной статьи.

Участие авторов. Все авторы внесли существенный вклад в проведение поисково-аналитической работы и подготовку статьи, прочли и одобрили итоговую версию до публикации.

\section{СПИСОК ЛИТЕРАТУРЫ | REFERENCES}

1. Melmed S. Acromegaly. N Eng/ J Med. 1990;322(14):966-977. doi: https://doi.org/10.1056/NEJM199004053221405

2. Biermasz NR, Pereira AM, Smit JW, et al. Morbidity after long-term remission for acromegaly: persisting joint-related complaints cause reduced quality of life. J Clin Endocrinol Metab. 2005;90(5):2731-2739. doi: https://doi.org/10.1210/jc.2004-2297

3. Ben-Shlomo A, Melmed S. Acromegaly. Endocrinol Metab Clin North Am. 2001;30(3):565-583. doi: https://doi.org/10.1016/s0889-8529(05)70202-4

4. Detenbeck LC, Tressler HA, O'Duffy JD, Randall RV. Peripheral joint manifestations of acromegaly. Clin Orthop Relat Res. 1973;(91):119-127. doi: https://doi.org/10.1097/00003086-197303000-00017

5. Killinger Z, Payer J, Lazurova I, et al. Arthropathy in acromegaly. Rheum Dis Clin North Am. 2010;36(4):713-720. doi: https://doi.org/10.1016/j.rdc.2010.09.004

6. Wassenaar MJ, Biermasz NR, van Duinen N, et al. High prevalence of arthropathy, according to the definitions of radiological and clinical osteoarthritis, in patients with long-term cure of acromegaly: a case-control study. Eur J Endocrinol. 2009;160(3):357-365. doi: https://doi.org/10.1530/EJE-08-0845

7. Barkan AL. Acromegalic arthropathy. Pituitary. 2001;4(4):263-264. doi: https://doi.org/10.1023/a:1020754615863

8. Scarpa R, De Brasi D, Pivonello R, et al. Acromegalic axial arthropathy: a clinical case-control study. J Clin Endocrinol Metab. 2004;89(2):598-603. doi: https://doi.org/10.1210/jc.2003-031283
9. Wassenaar MJ, Biermasz NR, Pereira AM, et al. The exon-3 deleted growth hormone receptor polymorphism predisposes to long-term complications of acromegaly. J Clin Endocrinol Metab. 2009;94(12):4671-4678. doi: https://doi.org/10.1210/jc.2009-1172

10. Fortier LA, Mohammed HO, Lust G, Nixon AJ. Insulin-like growth factor-I enhances cell-based repair of articular cartilage. J Bone Joint Surg Br. 2002;84(2):276-288. doi: https://doi.org/10.1302/0301-620x.84b2.11167

11. Miller RE, Grodzinsky AJ, Cummings K, et al. Intraarticular injection of heparin-binding insulin-like growth factor 1 sustains delivery of insulin-like growth factor 1 to cartilage through binding to chondroitin sulfate. Arthritis Rheum. 2010;62(12):3686-3694. doi: https://doi.org/10.1002/art.27709

12. Barkan A. Acromegalic arthropathy and sleep apnea. J Endocrinol. 1997;155 Suppl 1:S41-S44.

13. Chipman JJ, Attanasio AF, Birkett MA, et al. The safety profile of GH replacement therapy in adults. Clin Endocrinol (Oxf). 1997;46(4):473-481. doi: https://doi.org/10.1046/j.1365-2265.1997.1660984.x

14. Colao A, Cannavo S, Marzullo P, et al. Twelve months of treatment with octreotide-LAR reduces joint thickness in acromegaly. Eur J Endocrinol. 2003;148(1):31-38. doi: https://doi.org/10.1530/eje.0.1480031

15. Wassenaar MJ, Biermasz NR, Bijsterbosch J, et al. Arthropathy in long-term cured acromegaly is characterised by osteophytes without joint space narrowing: a comparison with generalised osteoarthritis. Ann Rheum Dis. 2011;70(2):320-325. doi: https://doi.org/10.1136/ard.2010.131698 
16. Claessen KM, Kroon HM, Pereira AM, et al. Progression of vertebral fractures despite long-term biochemical control of acromegaly: a prospective follow-up study. J Clin Endocrinol Metab. 2013;98(12):4808-4815. doi: https://doi.org/10.1210/jc.2013-2695

17. Kropf LL, Madeira M, Vieira Neto L, et al. Functional evaluation of the joints in acromegalic patients and associated factors. Clin Rheumatol. 2013;32(7):991-998. doi: https://doi.org/10.1007/s10067-013-2219-1

18. Мокрышева Н.Г., Еремкина А.К., Мирная С.С., и др. Патологические изменения в суставах и мышцах при первичном гиперпаратиреозе. // Остеопороз и остеопатии. - 2018. T. 21. - №4. - C. 10-18. [Mokrysheva NG, Eremkina AK, Mirnaya SS, et al. Joint and muscle involvement in primary hyperparathyroidism. Osteoporosis and bone diseases. 2018;21(4):10-18. (In Russ.)] doi: https://doi.org/10.14341/osteo9783

19. Claessen K, Canete AN, de Bruin PW, et al. Acromegalic arthropathy in various stages of the disease: an MRI study. Eur J Endocrinol. 2017;176(6):779-790. doi: https://doi.org/10.1530/EJE-16-1073

20. Bluestone R, Bywaters EG, Hartog M, et al. Acromegalic arthropathy. Ann Rheum Dis. 1971;30(3):243-258. doi: https://doi.org/10.1136/ard.30.3.243

21. Nezu M, Kudo M, Morimoto R, et al. Effects of surgical treatment for acromegaly on knee MRI structural features. Endocr J. 2018;65(10):991-999. doi: https://doi.org/10.1507/endocrj.EJ18-0108

22. Wassenaar MJ, Biermasz NR, Kloppenburg M, et al. Clinical osteoarthritis predicts physical and psychological QoL in acromegaly patients. Growth Horm IGF Res. 2010;20(3):226-233. doi: https://doi.org/10.1016/j.ghir.2010.02.003

23. Claessen KM, Kloppenburg M, Kroon HM, et al. Two phenotypes of arthropathy in long-term controlled acromegaly? A comparison between patients with and without joint space narrowing (JSN). Growth Horm IGF Res. 2013;23(5):159-164. doi: https://doi.org/10.1016/j.ghir.2013.05.003

24. Felson DT, Lawrence RC, Dieppe PA, et al. Osteoarthritis: new insights. Part 1: the disease and its risk factors. Ann Intern Med. 2000;133(8):635-646. doi: https://doi.org/10.7326/0003-4819-133-8-200010170-00016

25. Biermasz NR, Pereira AM, Frolich $M$, et al. Octreotide represses secretory-burst mass and nonpulsatile secretion but does not restore event frequency or orderly GH secretion in acromegaly. Am J Physiol Endocrinol Metab. 2004;286(1):E25-30. doi: https://doi.org/10.1152/ajpendo.00230.2003

26. van Thiel SW, Bax JJ, Biermasz NR, et al. Persistent diastolic dysfunction despite successful long-term octreotide treatment in acromegaly. Eur J Endocrinol. 2005;153(2):231-238. doi: https://doi.org/10.1530/eje.1.01955

27. Weiss RE, Reddi AH, Nimni ME. Somatostatin can locally inhibit proliferation and differentiation of cartilage and bone precursor cells. Calcif Tissue Int. 1981;33(4):425-430. doi: https://doi.org/10.1007/bf02409466

28. Zapf J, Gosteli-Peter M, Weckbecker G, et al. The somatostatin analog octreotide inhibits $\mathrm{GH}$ stimulated, but not IGF-I-stimulated, bone growth in hypophysectomized rats. Endocrinology. 2002;143(8):2944-2952. doi: https://doi.org/10.1210/endo.143.8.8970

29. Sievers C, Brubach K, Saller B, et al. Change of symptoms and perceived health in acromegalic patients on pegvisomant therapy: a retrospective cohort study within the German Pegvisomant Observational Study (GPOS). Clin Endocrinol (Oxf). 2010;73(1):89-94. doi: https://doi.org/10.1111/j.1365-2265.2009.03773.x

30. Neggers SJ, van Aken MO, de Herder WW, et al. Quality of life in acromegalic patients during long-term somatostatin analog treatment with and without pegvisomant. J Clin Endocrinol Metab. 2008;93(10):3853-3859. doi: https://doi.org/10.1210/jc.2008-0669

31. Colao A, Marzullo P, Vallone G, et al. Reversibility of joint thickening in acromegalic patients: an ultrasonography study. J Clin Endocrinol Metab. 1998;83(6):2121-2125. doi: https://doi.org/10.1210/jcem.83.6.4865

32. Colao A, Marzullo P, Vallone G, et al. Ultrasonographic evidence of joint thickening reversibility in acromegalic patients treated with lanreotide for 12 months. Clin Endocrinol (Oxf). 1999;51 (5):611-618. doi: https://doi.org/10.1046/j.1365-2265.1999.00851.x

33. Claessen KM, Ramautar SR, Pereira AM, et al. Progression of acromegalic arthropathy despite long-term biochemical control: a prospective, radiological study. Eur J Endocrinol. 2012;167(2):235244. doi: https://doi.org/10.1530/EJE-12-0147
34. Neggers SJ, van Aken $\mathrm{MO}$, de Herder WW, et al. Quality of life in acromegalic patients during long-term somatostatin analog treatment with and without pegvisomant. J Clin Endocrinol Metab. 2008;93(10):3853-3859. doi: https://doi.org/10.1210/jc.2008-0669

35. Podgorski M, Robinson B, Weissberger A, et al. Articular manifestations of acromegaly. Aust N Z J Med. 1988;18(1):28-35. doi: https://doi.org/10.1111/j.1445-5994.1988.tb02236.x

36. Colao A, Ferone D, Marzullo P, Lombardi G. Systemic complications of acromegaly: epidemiology, pathogenesis, and management. Endocr Rev. 2004;25(1):102-152. doi: https://doi.org/10.1210/er.2002-0022

37. Colao A, Pivonello R, Scarpa R, et al. The acromegalic arthropathy. J Endocrinol Invest. 2005;28(8 Suppl):24-31.

38. Lacks S, Jacobs RP. Acromegalic arthropathy: a reversible rheumatic disease. J Rheumatol. 1986;13(3):634-636.

39. Mazziotti G, Bianchi A, Bonadonna S, et al. Prevalence of vertebral fractures in men with acromegaly. J Clin Endocrinol Metab. 2008;93(12):4649-4655. doi: https://doi.org/10.1210/jc.2008-0791

40. Romijn JA. Acromegalic arthropathy: current perspectives. Endocrine. 2013:43(2):245-246. doi: https://doi.org/10.1007/s12020-012-9781-1

41. Mazziotti G, Bianchi A, Porcelli T, et al. Vertebral Fractures in Patients With Acromegaly: A 3-Year Prospective Study. J Clin Endocrinol Metab. 2013;98(8):3402-3410. doi: https://doi.org/10.1210/jc.2013-1460

42. Padova G, Borzi G, Incorvaia L, et al. Prevalence of osteoporosis and vertebral fractures in acromegalic patients. Clin Cases Miner Bone Metab. 2011;8(3):37-43.

43. Mazziotti G, Gola M, Bianchi A, et al. Influence of diabetes mellitus on vertebral fractures in men with acromegaly. Endocrine. 2011;40(1):102-108. doi: https://doi.org/10.1007/s12020-011-9486-x

44. Madeira M, Neto LV, de Paula Paranhos Neto F, et al. Acromegaly Has a Negative Influence on Trabecular Bone, But Not on Cortical Bone, as Assessed by High-Resolution Periphera Quantitative Computed Tomography. J Clin Endocrinol Metab. 2013;98(4):1734-1741. doi: https://doi.org/10.1210/jc.2012-4073

45. Wassenaar MJE, Biermasz NR, Hamdy NAT, et al. High prevalence of vertebral fractures despite normal bone mineral density in patients with long-term controlled acromegaly. Eur J Endocrinol. 2011;164(4):475-483. doi: https://doi.org/10.1530/eje-10-1005

46. Lesse GP, Fraser WD, Farquharson $\mathrm{R}$, et al. Gonadal status is an important determinant of bone density in acromegaly. Clin Endocrinol (Oxf). 1998;48(1):59-65. doi: https://doi.org/10.1046/j.1365-2265.1998.00349.x

47. Mazziotti G, Bianchi A, Bonadonna S, et al. Increased prevalence of radiological spinal deformities in adult patients with $\mathrm{GH}$ deficiency: influence of GH replacement therapy. J Bone Miner Res. 2006;21(4):520-528. doi: https://doi.org/10.1359/jbmr.060112

48. Genant HK, Wu CY, van Kuijk C, Nevitt MC. Vertebral fracture assessment using a semiquantitative technique. J Bone Miner Res. 1993;8(9):1137-1148. doi: https://doi.org/10.1002/jbmr.5650080915

49. Oruk G, Tarhan F, Argin M, Ozmen M. Is every joint symptom related to acromegaly? Endocrine. 2013;43(2):404-411. doi: https://doi.org/10.1007/s12020-012-9770-4

50. Ozcakar L, Akinci A, Bal S. A challenging case of rheumatoid arthritis in an acromegalic patient. Rheumatol Int. 2003;23(3):146-148. doi: https://doi.org/10.1007/s00296-002-0280-1

51. Aydin $Y$, Coskun H, Kir S, et al. Rheumatoid arthritis masquerading as acromegaly recurrence: report of two cases. Rheumatol Int. 2012;32(9):2913-2915. doi: https://doi.org/10.1007/s00296-010-1490-6

52. Read RC, Watt I, Dieppe PA. Rheumatoid arthritis and acromegaly-effect of growth factors on RA? Br J Rheumatol. 1988;27(3):242-243. doi: https://doi.org/10.1093/rheumatology/27.3.242

53. Miyoshi T, Otsuka F, Kawabata T, et al. Manifestation of rheumatoid arthritis after transsphenoidal surgery in a patient with acromegaly. Endocr J. 2006;53(5):621-625. doi: https://doi.org/10.1507/endocrj.k06-043

54. Nachtigall LB. Acromegaly diagnosed in a young woman presenting with headache and arthritis. Nat Clin Pract Endocrinol Metab. 2006;2(10):582-587. doi: https://doi.org/10.1038/ncpendmet0301

55. Chanson P, Salenave S, Kamenicky P, et al. Pituitary tumours: acromegaly. Best Pract Res Clin Endocrinol Metab. 2009;23(5):555-574. doi: https://doi.org/10.1016/j.beem.2009.05.010 
56. Паневин Т.С., Алекперов Р.Т., Мельниченко Г.А. Синдром Рейно в практике эндокринолога. // Ожирение и метаболизм — 2019. - T. 16. — №4. (в печати) [Panevin TS, Alekperov RT, Melnichenko GA. Raynaud's phenomenon in the endocrinologist's practice. Obesity and metabolism. 2019;16(4) (in press). (In Russ.)] doi: https://doi.org/https://doi.org/10.14341/omet10245

57. Schiavon F, Maffei P, Martini C, et al. Morphologic study of microcirculation in acromegaly by capillaroscopy. J Clin Endocrinol Metab. 1999:84(9):3151-3155. doi: https://doi.org/10.1210/jcem.84.9.5952
58. Miller A, Doll H, David J, Wass J. Impact of musculoskeletal disease on quality of life in longstanding acromegaly. Eur J Endocrinol. 2008;158(5):587-593. doi: https://doi.org/10.1530/EJE-07-0838

59. Kameyama S, Tanaka R, Hasegawa A, et al. Subclinical carpal tunnel syndrome in acromegaly. Neurol Med Chir (Tokyo). 1993;33(8):547-551. doi: https://doi.org/10.2176/nmc.33.547

60. Tani Y, Tanaka N, Isoya E. Locking of metacarpophalangeal jointsin a patient with acromegaly. Skeletal Radiol.1999;28(11):655-657. doi: https://doi.org/10.1007/s002560050569

\section{ИНФОРМАЦИЯ ОБ АВТОРАХ [AUTHORS INFO]}

* Паневин Тарас Сергеевич [Taras S. Panevin, MD]; адрес: Россия, 117036, Москва, ул. Дм. Ульянова, д. 11 [address: 11 Dm. Ulyanova street, 117036 Moscow, Russia]; Россия, 115522, Москва, Каширское шоссе, д.34А [address: 34A Kashirskoe Shosse, 115522 Moscow, Russia]; ORCID: http://orcid.org/0000-0002-5290-156X; eLibrary SPIN-код: 7839-3145; e-mail: tarasel@list.ru

Алексеева Людмила Ивановна, д.М.н. [Lyudmila I. Alekseeva, MD, PhD]; ORCID: https://orcid.org/0000-0001-7017-0898; eLibrary SPIN: 4714-8572; e-mail: dr.alekseeva@gmail.com Мельниченко Галина Афанасьевна, д.м.н., профессор, академик PAH [Galina A. Melnichenko, MD, PhD, Professor]; ORCID: http://orcid.org/0000-0002-5634-7877; eLibrary SPIN: 8615-0038; e-mail: teofrast2000@mail.ru

\section{ЦИТИРОВАТЬ:}

Паневин Т.С., Алексеева Л.И., Мельниченко Г.А. Ревматические проявления акромегалии // Остеопороз и остеопатии. 2019. — Т. 22. — №2. - C.14-22. doi: https://doi.org/10.14341/osteo11353

\section{TO CITE THIS ARTICLE:}

Panevin TS, Alekseeva LI, Melnichenko GA. Rheumatic manifestations of acromegaly. Osteoporosis and bone diseases. 2019;22(2):14-22. doi: https://doi.org/10.14341/osteo11353 